\title{
Facile chemical strategy to synthesize Ag@ polypyrrole microarrays and investigating its anisotropic effect on polymer conductivity
}

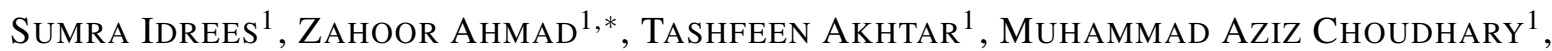 \\ Muhammad Aftab RafiQ ${ }^{2}$, Arshad Mehmood ${ }^{3}$ \\ ${ }^{1}$ Department of Chemistry, Mirpur University of Science and Technology (MUST), Allama Iqbal Road, Mirpur, AJ\&K, \\ Pakistan \\ ${ }^{2}$ Department of Physics and Applied Mathematics, Pakistan Institute of Engineering and Applied Sciences (PIEAS) \\ Islamabad, Pakistan \\ ${ }^{3}$ Material Science and Engineering Division, National Institute of Laser and Optronics (NILOP), PIEAS, Nilore, Islamabad
}

A facile chemical approach was developed to fabricate microarrays (MAs) of Ag@ polypyrrole nanocables (NCs). The strategy involved crosslinking the NCs by tetraethoxy silane (TEOS) under continuous pulse sonication without using a substrate. The material was characterized by scanning electron microscope (SEM) coupled with EDX, which revealed the longitudinal interconnections within the nanocables and creating unidirectional alignment in the form of MAs. FT-IR and Raman spectroscopy was employed to characterize the encapsulating polymer as polypyrrole (ppy) around Ag nanowires (NWs). The microarrays produced red shift in surface plasmon resonance (SPR) of Ag NWs, and drastically improved the thermal stability and conductivity of encasing ppy. It has imparted anisotropic conductivity effect on ppy which resulted in sharp decrease in resistivity from $8.35 \times 10^{10} \Omega$ to $2.449 \Omega$, when NCs were isolated and crosslinked into MAs form, respectively. The drastic decrease in resistivity of ppy was due to the anisotropic effect produced by the MAs format of NWs.

Keywords: nano-assembly; NCs microarrays; thermal stability; anisotropic conductivity

\section{Introduction}

Fabricating the assembly of 1D nanostructures in microarrays form is core research in nanotechnology. It is an important configuration due to potential applications in electronics, photonics, and sensing devices [1]. It involves MAs of DNA and organic supramolecules which find applications in biomedical nanodevices for medical diagnosis and drug delivery [2-5]. Moreover, MAs of carbon nanotubes, $\mathrm{Si}$ nanowires, and $\mathrm{ZnO}$ hexagonal nanorods were also explored and reported for multiple applications in electronics, and photovoltaic cells $[6,7]$. The fabrication of MAs was usually performed using substrates, such as anodic aluminum oxide, mesoporous silica, and cobalt

\footnotetext{
*E-mail: zahoor.chem@must.edu.pk; zahoor.chem@hotmail.com
}

structures [1, 8-10], applying technique of molecular self-assembly [2], dielectrophoretic forces [9], and laser or $\mathrm{N}_{2}$ gas pulses [11-13].

Herein, we report a facile strategy to fabricate the MAs of metal based NCs, which has been exploited for Ag@ppy system. It includes polyol synthesis of Ag NWs, cations mediated production of Ag@ppy NCs, and finally crosslinking with TEOS under pulse sonication. The simple pulse sonication oriented the NCs longitudinally and TEOS interlinked them into a stable MAs configuration. The mechanism of pulse sonication consisted in producing torque to $\mathrm{NCs}$ and aligning them in the pulse wave direction. The as-produced MAs were characterized by microscopic and spectroscopic techniques. The material demonstrated red shift in SPR and increase in thermal stability for encasing polymer. In addition, the material showed a sharp rise in conductivity of encapsulating 
polymer ppy due to the anisotropic effect of enclosed Ag NWs. So designed strategy is quite simple and applicable to developing the MAs of different densities, constituting few to several NCs according to the particular requirements for internal circuit units in nanodevices. It could be used as microchips for sensing and diagnosing biological target molecules by functionalizing their surface polymer.

\section{Materials and method}

\subsection{Reagents}

Ethylene glycol $99 \%$ (Sigma Aldrich), silver nitrate $99.9 \%$ (analytical reagent, Sigma Aldrich), PVP (Daejung Reagent Chemicals Korea), $95 \%$ ethanol, acetone, methanol (Sigma Aldrich), copper(1l)acetate monohydrate (Riedel-de Haen), pyrrole $98 \%$ (BDH Chemicals LTD), acetic acid (Sigma Aldrich $99.7 \%$ ), tetraethoxy silane (TEOS, $98 \%$ ) Merck, Germany were used in the experiments.

\subsection{Methods}

The microarrays of $\mathrm{Ag} @$ ppy NCs were produced in four simple steps. In step 1, Ag nanowires (NWs) were synthesized by polyol reduction method as reported in the literature [14]. $\mathrm{AgNO}_{3}$ (10 mL; $0.004 \mathrm{M})$ and PVP (0.006 M) were separately dissolved in ethylene glycol (EG) and added dropwise into preheated $15 \mathrm{~mL}$ EG. The temperature was maintained at $160{ }^{\circ} \mathrm{C}$ and addition of $\mathrm{AgNO}_{3}$ was started to generate the growth of $\mathrm{Ag}$ NWs seeds. The reaction was allowed to continue for one hour at the same temperature. It produced gray material that was washed with acetone and ethanol, and centrifuged several times at $3000 \mathrm{rpm}$ to remove EG and PVP.

In the second step, the as-obtained NWs were coated with ppy using ion adsorption method also reported in the literature [15], which produced $\mathrm{Ag} @$ ppy NCs. The reaction was allowed to proceed for 5 hours in methanol for completion. The NCs were again washed with ethanol and centrifuged at the same speed to remove unreacted monomer and oxidizing agent.
The third step consisted in crosslinking of Ag@ppy NCs in $10 \%, 50 \mu \mathrm{L}$ acetic acid solution by adding $100 \mu \mathrm{L}$ TEOS as the crosslinking agent. The crosslinking reaction was continued for $5 \mathrm{~h}$ to $6 \mathrm{~h}$ under conventional stirring, where $\mathrm{Ag} @$ ppy NCs randomly linked with each other. Finally, the same experiment was performed under continuous pulse sonication, which produced torque to NCs and aligned them unidirectionally. The crosslinking agent (TEOS) crosslinked the unidirectional NCs into a stable MAs configuration. Thus, a simple physicochemical approach was developed, which proved helpful to fabricate stable microarrays of Ag@ppy NCs. Such structure is constituted by NCs rather than NWs where bare metal nanostructures could merge with each other into nanoagglomerates and lose their metamaterials characteristics. The as-fabricated microarrays of Ag@ppy NCs were characterized and their important properties were investigated.

\subsection{Characterization}

The morphology and material were characterized by FE-SEM TESCAN MIRA3XMU, SEM coupled with EDS system. Polymer coating was confirmed by Fourier transform infra-red (FT-IR) and Raman spectroscopy, which were recorded on Nicolet iS10 FT-IR and MST-4000 A (Germany) Raman instrument, respectively. The He-Cd laser with $532 \mathrm{~nm}$ wavelength was used in Raman scattering spectroscopy. Shimadzu-1800 UV-Vis spectrophotometer was employed to measure the SPR of isolated Ag NWs, NCs and their MAs form. Thermal stability of ppy over separated, randomly crosslinked NCs, and in MAs configuration of Ag NCs was determined using TGA Q50 instrument. Similarly, the conductivity of ppy over separated, randomly connected NCs and in MAs configuration of NCs was measured by Keithley 2400 $\mathrm{I}-\mathrm{V}$ instrument.

\section{Results and discussion}

The morphology of the microarrays developed by our own method observed under SEM has been displayed in Fig. 1. It shows pristine Ag NWs, Ag@ppy NCs, randomly crosslinked NCs and their 
microarrays format, respectively. The diameters of NWs and NCs shown in Fig. 1a and Fig. 1b were found to range from $80 \mathrm{~nm}$ to $100 \mathrm{~nm}$ and their length was within few microns. Fig. 1b clearly distinguishes ppy smooth coating over NWs. The random crosslinking produced by TEOS under ordinary stirring is reflected in Fig. 1c, where NCs appear as linked with each other. Such SEM observation encouraged us to believe that TEOS is a suitable crosslinking agent of NCs and it can transform the NCs into gel with pore size distribution ranging from few $\mathrm{nm}$ to few $\mu \mathrm{m}$. The gel of NCs is beneficial for a variety of applications like monolith material to develop stationary phase in HPLC and capillary electrophoresis chromatography [16]. Its surface can also act as adsorbent, sensor and agent for drug delivery [17]. The monolithic character can be further improved if the random crosslinking could be transformed into microarrays form. Therefore, an experiment was performed where pulse sonication was used along with TEOS as crosslinking agent. The results of this experiment are shown in Fig. $1\left(\mathrm{~d}_{\mathrm{I}}\right.$ and $\left.\mathrm{d}_{\mathrm{II}}\right)$. The Ag NCs transformed into MAs form that additionally demonstrated nano/micro channels, which may find numerous applications as described in the literature [16, 17]. Moreover, such MAs exhibit anisotropic character being reinforced within polymer coating. It may be useful in fast-response electrochromic displays and other nanodevices of Hi-Tech electronics.

The final product in the form of MAs, which had a structure of fine gel, might enhance the anisotropic character of NWs, which was further confirmed by EDS. The EDS data is given in Fig. 2 and Table 1.

Table 1. EDS determined elements within microarrays of nanocables.

\begin{tabular}{ccc}
\hline Element & wt.\% & at.\% \\
\hline \hline C K & 53.09 & 63.41 \\
N K & 21.40 & 21.92 \\
O K & 14.38 & 12.89 \\
Si K & 0.63 & 0.32 \\
Cu K & 0.66 & 0.15 \\
Ag L & 9.84 & 1.31 \\
\hline
\end{tabular}

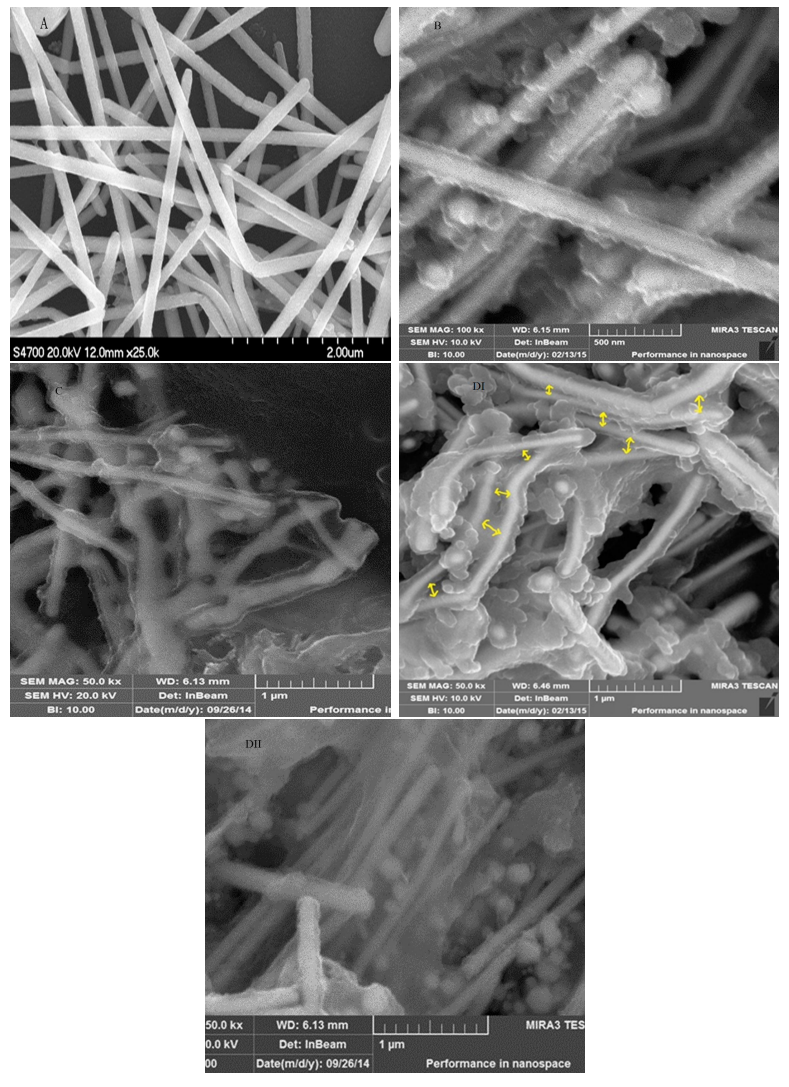

Fig. 1. (a) SEM micrographs of NWs, (b) NCs, (c) randomly interlinked gel of NCs, and (d) longitudinally interlinked NCs making microarrays of different densities $d_{I}$ and $d_{I I}$, respectively, with smaller pore size and micro/nanochannels.

The EDS spectrum, displayed in Fig. 2, and the respective Table 1, clearly show the elemental composition of Ag@ppy MAs. According to the data, $\mathrm{Ag}$ belongs to NWs, $\mathrm{Cu}$ is present as ion adsorbate, $\mathrm{C}$ and $\mathrm{N}$ from polymer (ppy), and $\mathrm{Si}$ and $\mathrm{O}$ belong to TEOS, which developed stable longitudinal linkages during the pulse sonication to fabricate the microarrays from NCs.

The elemental mapping of MAs further supports the EDS results. It is displayed in Fig. 3, which clearly shows the distribution of elements, such as carbon, oxygen, silicon, and silver. It reflects the existence of Si between the polymers chains, which come from TEOS and covalently configured NCs into MAs form.

The ppy component of the microarrays was further characterized by FT-IR and Raman 


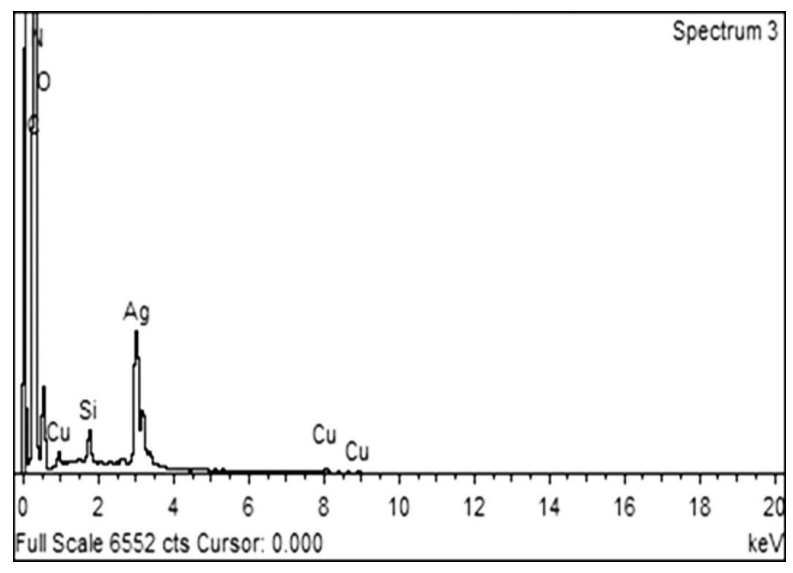

Fig. 2. EDS pattern of longitudinally interlinked NCs making microarrays.

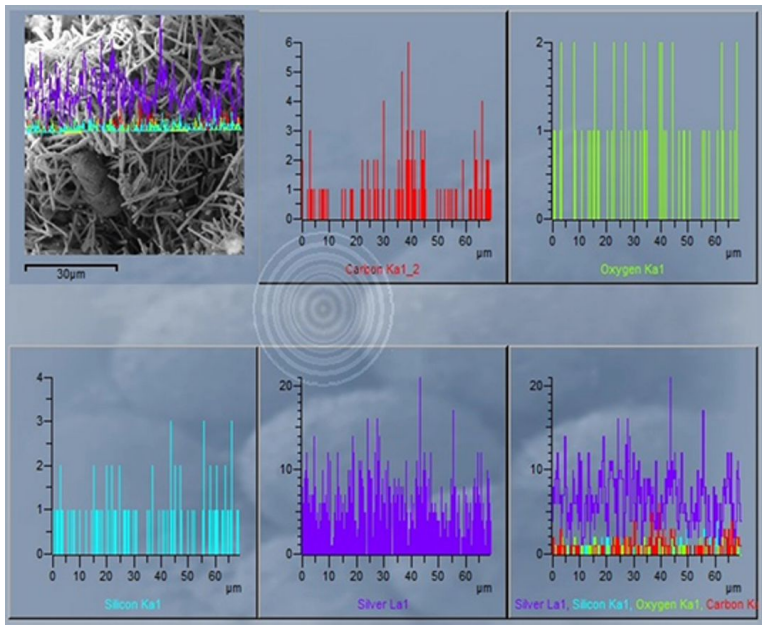

Fig. 3. Elemental mapping of microarrays of Ag@ppy NCs.

spectroscopy. The FT-IR and Raman spectra are shown in Fig. 4a and Fig. 4b, respectively. According to the FT-IR spectra, all the bands are very broad, which is typical of polymer material. The band at $3500 \mathrm{~cm}^{-1}$ is attributed to $\mathrm{N}-\mathrm{H}$ stretching vibrations. The greater broadness of this band may be connected with some sort of hydrogen bonding or proton ionization due to close vicinity of intact plasma of assembled NWs. Other band that appeared at $2810 \mathrm{~cm}^{-1}$ is the characteristic vibration of tetriary amine, mixed with ppy $\mathrm{C}-\mathrm{H}$ vibrations [18, 19]. The bands at $1510 \mathrm{~cm}^{-1}$ and $1345 \mathrm{~cm}^{-1}$ are due to ppy ring and $\mathrm{C}-\mathrm{N}$ stretching vibrations [20]. The bands at $1195 \mathrm{~cm}^{-1}$ and $1065 \mathrm{~cm}^{-1}$ are attributed to $\mathrm{N}-\mathrm{H}$ and $\mathrm{C}-\mathrm{H}$ in plane bending mode (Ag ppy JNR) [15]. Furthermore, the $1065 \mathrm{~cm}^{-1}$ band may also be attributed to $\mathrm{N}-\mathrm{Si}$ bending [21].

Similarly, the Raman spectrum displayed in Fig. 4b, reveals ppy as surface constituting organic material. The prominent Raman shift which is centered at $1330 \mathrm{~cm}^{-1}$ belongs to stretching mode of pyrrole ring. Similarly, the other prominent Raman shift located at $1550 \mathrm{~cm}^{-1}$, typically attributed to $\mathrm{C}=\mathrm{C}$ backbone stretching, further manifests the presence of long range backbone conjugation [22]. Therefore, FT-IR and Raman spectroscopy evidenced the polymer material as ppy, which is beneficial for many other purposeful functionalization.

UV-Vis spectroscopy was employed to study the effect of polymer on surface plasmon resonance of Ag NWs, which have been fabricated in different formats. The comparative data is displayed in Fig. 5. The pure Ag NWs demonstrate longitudinal and transverse SPR at $410 \mathrm{~nm}$ and $350 \mathrm{~nm}$ which is in compliance with the characteristics of 1D nano Ag as reported in the literature [23-25]. Thin coating of Ag NWs with ppy has greatly decreased the intensity of longitudinal SPR which evidences that ppy coating has not destroyed metal SPR, but relatively hindered some surface plasmon interaction with electromagnetic radiation. However, when these NCs were configured into MAs form, the typical SPR of Ag NWs disappeared and seemed remarkably red shifted. The long range shifting was primarily attributed to the anisotropic surface plasmon unification, which resulted in an increase in its magnitude that enabled it to interact with the light of low energy or light of longer wavelength. Thus, MAs configuration proved reliable to transfer surface plasmon wave from visible region toward NIR region.

To evaluate the effect of crosslinking in random and MAs configurations on thermal stability of polymer, TGA was recorded and displayed in Fig. 6. Usually ppy is considered as thermally stable polymer, which improves its thermal stability when some reinforcing material is used or it is crosslinked. According to another study pure and isolated ppy were found to lose the weight 

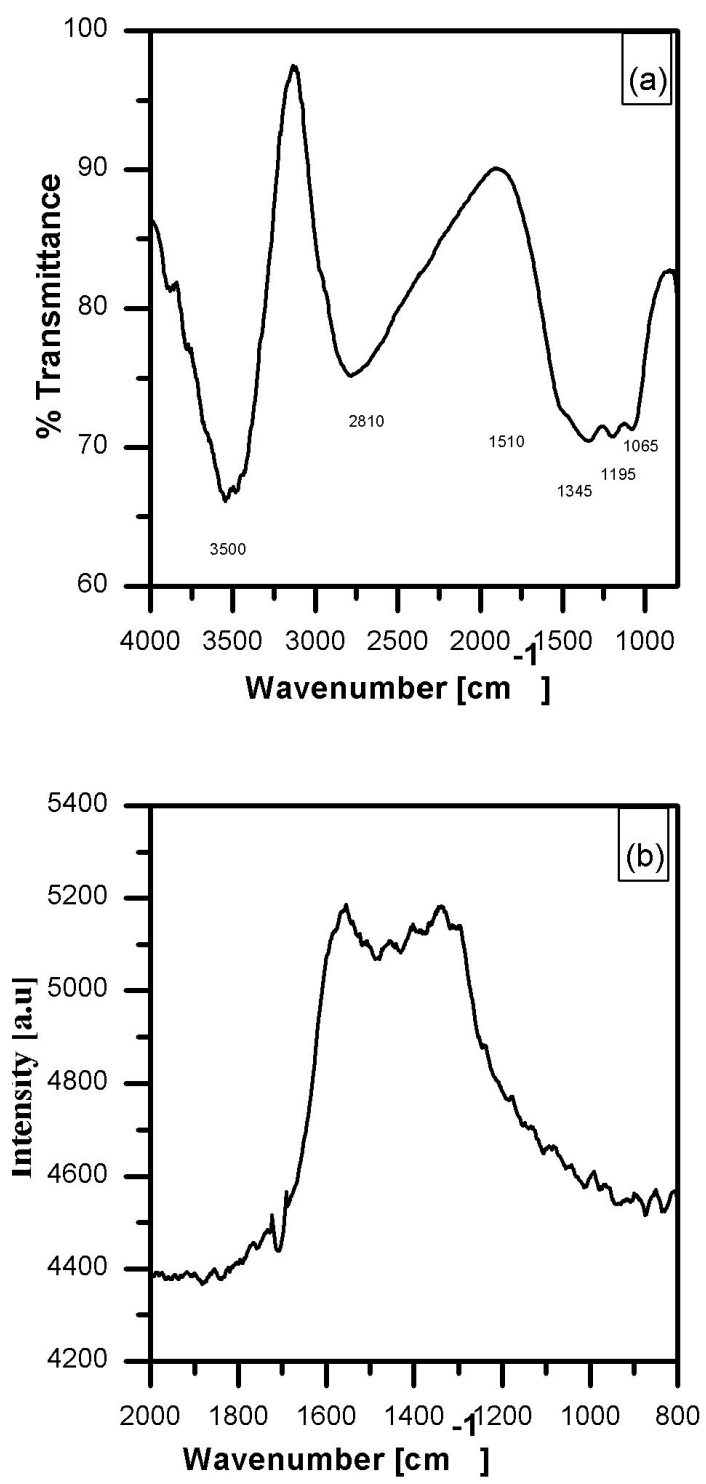

Fig. 4. FT-IR (a) and Raman spectrum (b) of Ag@ppy microarrays.

in the range of $200{ }^{\circ} \mathrm{C}$ to $220{ }^{\circ} \mathrm{C}$ [26], but here, in NCs form, it was found to be quite stable up to $300{ }^{\circ} \mathrm{C}$, which was the temperature where its weight loss started. The weight loss was found quite sharp. However, when these NCs were randomly crosslinked into gel form, the sharp weight loss changed into gradual weight loss. It might be due to crosslinking effect, where polymer stability has been increased. Herein, the uncrosslinked

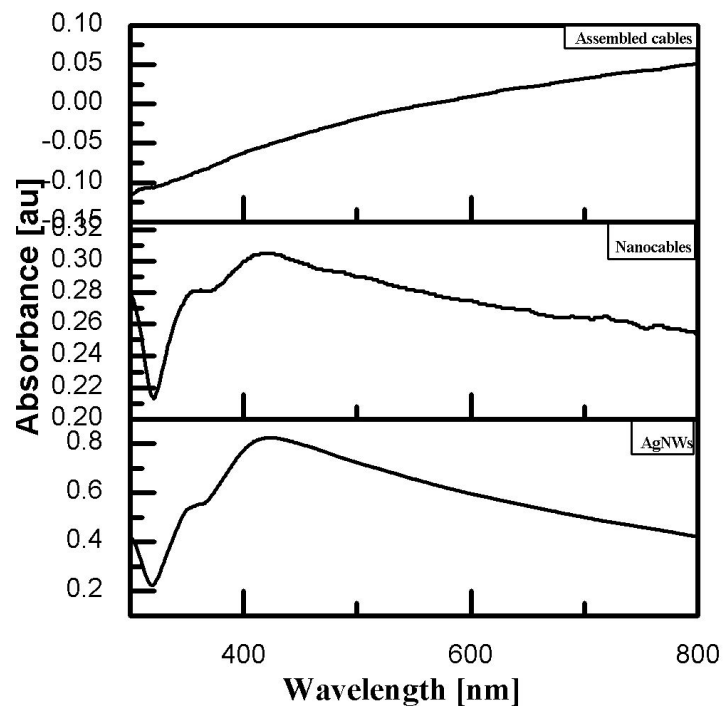

Fig. 5. UV-Vis spectra of Ag NWs, ppy coated Ag NCs and microarrays of Ag NCs.

fibers degraded first and crosslinked fibers gradually degraded depending upon the extent of their crosslinking. Finally, when these NCs were transformed into MAs form, ppy exhibited further stability and demonstrated more gradual weight loss. Therefore, in microarrays form, the polymer is sandwiched in longitudinally oriented NWs, where polymer internal attraction or its enthalpy further increased, which resulted in further improving its thermal stability. Such rise of thermal stability is quite consistent with reported data, where microarrays of CNT increased the thermal stability of different polymers like polystyrene or polyvinyl carbazole [24]. Therefore, the metal microarrays in reinforced form produced higher thermal stability in polymer as compared to its isolated form.

In addition, 1D metal nanostructures, like NWs, are quite suitable material to develop anisotropic behavior. They are appropriate to design single crystalline orientation [15]. With reference to conductivity, they confine de Broglie wave function of electron within its radius and allow its propagation along longitudinal domain [25]. Therefore, 1D metal nanostructures demonstrate anisotropic conductivity. Herein, the anisotropic 


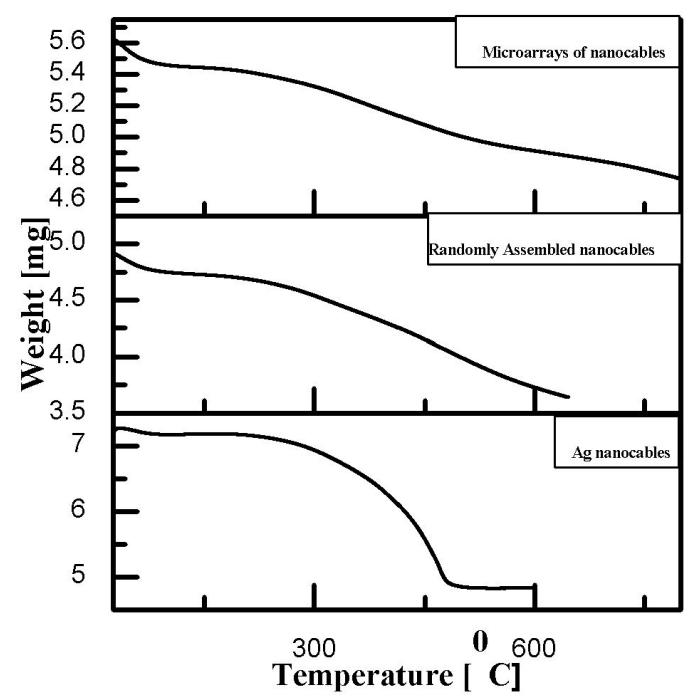

Fig. 6. TGA curves of ppy degradation when coated on Ag NWs, randomly crosslinked and crosslinked into microarrays format.

conductivity was investigated using Keithley 2400 $\mathrm{I}-\mathrm{V}$ instrument and the data is given in Fig. 7. The samples of Ag@ppy NCs, simple gel of NCs and MAs of NCs were pressed into pellets of few millimeter thickness. Four probe method was applied to measure the current in Amperes by applying the potential difference from $-10 \mathrm{~V}$ to $10 \mathrm{~V}$. Later on, resistivity was calculated using the relation given as:

$$
R=V / I
$$

The average resistivity of Ag@ppy NCs, simple gel of NCs and MAs configured NCs calculated from the data given in Fig. 7, is $8.35 \times 10^{10}$ $\Omega, 3.58 \times 10^{8} \Omega$ and $2.449 \Omega$, respectively. It was believed that current response to potential difference or resistivity depends on ppy that is a surface coating material. The average resistivity of ppy over isolated NCs is $8.35 \times 10^{10} \Omega$, which is quite high. It was found comparable to pure ppy which was prepared by the oxidative polymerization $[25,26]$. Such high resistivity demonstrated by ppy in isolated NCs shows that Ag NWs within polymer have not contributed to any extent. However, when NCs were randomly crosslinked into gel form as shown in Fig. 1c, ppy demonstrated comparatively lower resistivity which was
$3.58 \times 10^{8} \Omega$. It might be due to nanometal particles which got closer to each other and increased the current response due to decreased inbuilt resistivity. Similarly, the average resistivity of ppy over NCs microarrays was calculated. They demonstrated the resistivity of $2.449 \Omega$, which was comparatively less. Here, ppy revealed its highest current response and the lowest resistivity, when NCs were configured into MAs form, as shown in Fig. 1d. The greater current I and sharp decrease of resistivity were attributed to the unidirectional arrangement of $\mathrm{Ag}$ NWs within the microarrays of Ag NCs. The structural anisotropy enhanced the conductivity of enclosing ppy. Thus, I-V response in MAs form, supported the view that there was longitudinal propagation of electronic wave function in Ag NWs rather than radial, which is purely anisotropic in character. Therefore, using microarrays of NWs as reinforcing material could exponentially increase the conductivity of polymer that is quite resistive without such reinforcing material. The microarrays of NCs are considered as structural component of nanodevices in Hi-Tech electronics which can be used as junction material or interconnects. They can also be used as charge transporting agent in Li-ion batteries. Therefore, the developed strategy would be helpful to produce diversity of NCs based microarrays of different densities according to device requirement.

\section{Conclusions}

The chemical approach under pulse sonication has been proven successful to fabricate microarrays of Ag@ppy NCs. It combines the physical force which produces torque within NCs in a particular direction and then develops chemical interlinking to produce stable setting of microarrays. The as-fabricated microarrays were observed by SEM. The material was characterized by EDS, elemental mapping, FT-IR and Raman spectroscopy. The SPR, thermal behavior, and conductivity of the material in random and microarray form showed significantly improved trend. The produced MAs developed SPR of high amplitude and longer wavelength which can interact with light of lower energy. Similarly, MAs 


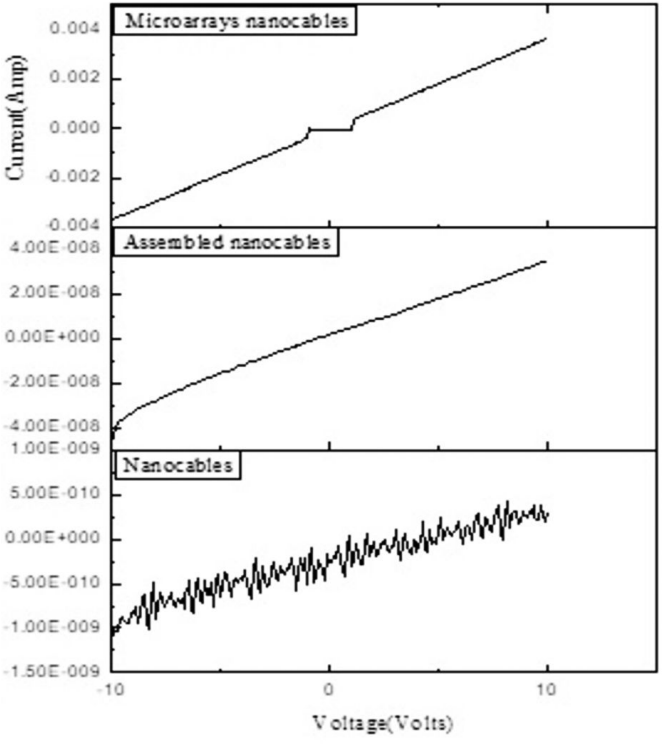

Fig. 7. Conductivity of ppy in pure nanocable, randomly assembled nanocables and orderly assembled (microarrays) nanocables.

improved the thermal stability of polymer. Finally, ppy in MAs form demonstrated exceptionally low resistivity that was $2.449 \Omega$, which in other cases was too high, i.e. $8.35 \times 10^{10} \Omega$, or $3.58 \times 10^{8}$ $\Omega$. The method can further be optimized to obtain different densities of MAs according to device requirements. The developed strategy may prove helpful to improve the mechanical behavior of any encapsulated polymer. It is a material that can be potentially used as interconnect in internal circuit units of nanodevices or charge transporting agent in $\mathrm{Li}$-ion batteries.

\section{Acknowledgements}

The Higher Education Commission of Pakistan is gratefully acknowledged due its funding to complete the work. Prof. Dr. Jameel Ahmad, department of Metallurgy and Materials Engineering, PIEAS, is highly acknowledged for his valuable efforts in assisting measurement of the conductivity of the materials.

\section{References}

[1] Huang Z., Shimizu T., Senz S., Zhang Z., Zhang X., Lee W., Geyer N., Gosele U., Nano Lett., 9(7) (2009), 2519.

[2] LI Y., CU Y.T.H., LuO D., Nat. Biotechnol., 23 (2005), 885.
[3] Lin C., LiU Y., YAN H., Nano Lett., 7 (2007), 507.

[4] Lee H. J., Kim S.E., Kwon I.K., Park C., Kim C., YAng J., LeE S.C., Chem. Commun., 46 (2010), 377.

[5] Li Y., Du W., Sun G., Wooley K. L., Macromol., 41 (2008), 6605.

[6] Ammu S., Heskett D., World J. Condens. Mat. Phys., 3 (2013), 159.

[7] Wang X., Summers C. J., Wang Z. L., Nano Lett., 4(3) (2004), 423.

[8] Feng X., Shankar K., Varghese O.K., Paulose M.V., LATEMPA T.J., GRIMES C.A., Nano Lett., 8(11) (2008), 3781.

[9] Gupta P., Rajput M., Singl N., Kumar, V., LAHIRI, D., Polymer, 89 (2016), 119.

[10] Yao B., Fleming D., Morris M.A., Lawrence S.E., Chem. Mater., 16 (2004), 4851.

[11] Son J.Y., Lim S.J., Cho J.H., SeOnG W.K., Kim H., Appl. Phy. Lett., 93 (5) (2008), 053109.

[12] Hill J.J., Haller K., Gelfand B., Ziegler K.J., ACS Appl. Mater. Interf., 2 (7) (2010), 1992.

[13] Jun T.X, Chao W.Y, Ning X.I., Li D.Z, Steve T., Chinese Sci. Bull., 53 (22) (2008), 3590.

[14] Ahmad Z., Teng Q., Jiangru Z., Xiaoyu L., J. Mater. Sci., 44 (2009), 6054.

[15] Teng Q., Xie H., Zhang J., Ahmad Z., Li X., J. Nanopart. Res., 13(3) (2010), 1175.

[16] Carn F., Colin A., Achard M.-F., Deleuze H., SAADi Z., BACKOV R., Adv. Mater., 16 (2004), 140.

[17] Jung J.H., PARK M., Shinkai S., Chem. Soc. Rev., 39 (2010), 4286.

[18] Ahmad Z., Choudhary M.A., Mirza M.A., Mirza J.A., Mater. Sci.-Poland, 34 (1) (2016), 79.

[19] Sundaraganesan N., Kalaichelvan S., Meganathan C., Spectrochim. Acta Part A, 71(2008), 898.

[20] Dominic J. B, Cornard, J., Sacchidanad S. S., JAYANT A. K., Milind V. K., Int. J. Innov. Res. Sci. Eng. Technol., 3(6) (2014), 14021.

[21] Huth F., Govyadinov A., Amarie S., NuansING W., Keilmann F., HillenbR R., Nano Lett., 12 (2012), 3973.

[22] Wengand Z., Ni X., J. Appl. Polym. Sci., 110 (2008), 109.

[23] Wang Z., LiU J., Chen X., Wan J., Qian Y., Chem. Eur. J., 11 (2005), 160.

[24] Yaozu L., Chen Z., Xia W. Xin-Gui L., Samuel J.I., Kourosh K., Phys. Chem. C., 115 (2011), 16187.

[25] Yugang S., Byron G., Brian M., Younan X., Nano Lett., 2 (2002), 165.

[26] Ahmad Z., Choudhary M.A. Mehmood A., WAKeEl R., Akhtar T., RAFIQ M.A., Macromol. Res., 24 (2016), 596. 